\title{
The Correlation Analysis on Glycolipid Metabolism, CysC and Homocysteine of Thyroid Dysfunction
}

\author{
Rongqin Dai ${ }^{1}$, Zhenjiang Hou ${ }^{1, ~ *}$, Jingyu Zhang ${ }^{2}, H_{0 n g}$ Fan $^{2}$, Hongsheng Wang ${ }^{2}$, Jianzhang Hou ${ }^{3}$ \\ ${ }^{1}$ Institute of Thyroid Diseases, Cangzhou Medical College, Cangzhou, China \\ ${ }^{2}$ Laboratory Diagnostics Division, Cangzhou Hospital of Integrated Traditional Chinese and Western Medicine, Cangzhou, China \\ ${ }^{3}$ Department of Surgery, Cangzhou Medical College Hospital District of Cangzhou People's Hospital, Cangzhou, China
}

Email address:

houzhenjiang@sina.com (Zhenjiang Hou)

${ }^{*}$ Corresponding author

\section{To cite this article:}

Rongqin Dai, Zhenjiang Hou, Jingyu Zhang, Hong Fan, Hongsheng Wang, Jianzhang Hou. The Correlation Analysis on Glycolipid Metabolism, CysC and Homocysteine of Thyroid Dysfunction. Science Journal of Public Health. Vol. 6, No. 2, 2018 , pp. 55-60. doi: $10.11648 /$ j.sjph.20180602.14

Received: December 9, 2017; Accepted: January 19, 2018; Published: February 5, 2018

\begin{abstract}
The objective is to discuss about the correlation of Glycolipid Metabolism, Cystatin C (CysC) and Homocysteine (Hcy) of thyroid dysfunction. 100 cases of hyperthyroidism, hypothyroidism and the control group are chose respectively to measure Fiber Bragg Grating (FBG), Blood Lipid, Thyroid Hormone (TH), CysC and Hcy, calculate the blood lipid ratio and make analysis. As a result, Thyroid Carcinoma (TC), Thyroglobulin (TG), Low-density Lipoprotein Cholesterol (LDL-C), High-density Lipoprotein Cholesterol (HDL-C), TC/HDL-C, TG/HDL-C, LDL-C/HDL-C, Lipid comprehensive index (LCI) and non-HDL-C of hyperthyroidism group are obviously lower than the control group. The decrease of LCI is the most prominent. Hcy is slightly lower than the control group. FBG and CysC are obviously higher than the control group. TC, TG, LDL-C, TG/HDL-C, LDL-C/HDL-C, LCI and non-HDL-C, Hcy of hypothyroidism group are higher than the control group $(\mathrm{P}<0.05)$, and $\mathrm{FBG}$, HDL-C and $\mathrm{Cys} C$ are lower than the control group $(\mathrm{P}<0.05)$. Various kinds of $\mathrm{TH}$ have the highest connection with TC, followed by non-HDL-C, LDL-C and LCI. It comes to the conclusion that patients with thyroid dysfunction easily appear the disorder of glucose and lipid metabolism. The change in blood lipid ratio is more pronounced than that of single lipid indicator and is closely related to CysC and Hcy. Dynamic monitoring of Thyroid Stimulating Hormone (TSH), blood lipid ratio, CysC and $\mathrm{Hcy}$ is of great value in the diagnosis of thyroid dysfunction and the prediction of Cardiovascular Disease (CVD) in concurrent Coronary Heart Disease (CHD) and so on.
\end{abstract}

Keywords: Hyperthyroidism, Hypothyroidism, Blood Glucose, Blood Lipid Ratio, Cystain C, Homocysteine

\section{Introduction}

The thyroid is the largest endocrine gland in the body. TH is secreted by thyroid, which plays an important regulatory role in the body sugar, fat and protein metabolism. In recent years, with changing in diet, increasing mental stress, accelerating pace of life, and increasing of environmental impact thyroid function material, the incidence of thyroid disease is on the rise. With the increase of the year, thyroid dysfunction and the positive rate of autoantibodies have significantly increased trend [1]. Thyroid dysfunction including hyperthyroidism and hypothyroidism, is caused by the abnormal secretion of $\mathrm{TH}$ syndrome, characterized by the increase or decrease of basic metabolism nerve excitability and induced disorder of sugar, lipid metabolism and other endocrine diseases. Cystatin $\mathrm{C}$ (CysC) and homocysteine (Hcy) can reflect the severity of coronary artery disease, which is closely related to the occurrence and development of coronary atherosclerosis (AS). Hypothyroidism with hyperhomocysteinemia indicates a higher prevalence of CVD, especially closely connect with coronary atherosclerosis (AS). Through the detection of thyroid dysfunction and the levels of blood glucose, blood lipids, CysC and Hcy of the control group, lipid ratio is calculated and done correlation analysis, which can provide a basis for the prevention and treatment of thyroid dysfunction and related diseases, now report as follows. 


\section{General Data and Methods}

\subsection{General Data}

A total of 200 patients with thyroid dysfunction visiting our hospital for health examination and our Endocrinology Dept. for treatment from April 2015 to December 2016, who were treated as the study subject. Among the subjects, there were 100 patients with hyperthyroidism: 36 males and 64 females, aged from 18 to 79 years old with mean age of (51.04 \pm 11.69$)$ years old; 100 patients with hypothyroidism: 28 males and 72 females, aged from 17 to 81 years old with mean age of $(52.36 \pm 14.36)$ years old and 100 healthy subjects taking health examination at the same period, were included as the control group: 40 males and 60 females aged from 21 to 81 years with mean age of $(48.17 \pm 13.52)$ years old. There was no significant difference in sex and age among the three groups $(\chi 2=2.979, \mathrm{P}=0.571 \mathrm{VS} \div 2=0.819, \mathrm{P}=0.526)$. The followings were excluded: with a history of liver and kidney diseases, diabetes, hypertension and CHD, pathoglycemia and dyslipidemia caused by other factors. And no medication that may impact thyroid functions and blood lipids were taken in the recent three months.

\subsection{Methods}

\subsubsection{Detection Methods}

All subjects were fasting for more than $12 \mathrm{~h}$, who was took 6 $\mathrm{ml}$ of venous blood during 7:30 to 9:30 in the morning and dispensed in 2 packs. The serum was separated centrifugally at $4000 \mathrm{r} / \mathrm{min}$ in time. TSH, Theban Tomb 3 (TT3), Theban Tomb (TT4), Free Triiodothyronine 3 (FT3) and Free Triiodothyronine 4 (FT4) were measured with Gobas E601 Immune Chemiluminescence Apparatus provided by German Roche Limited and agents were originally provided by Roche. FBG, TG, TC, HDL-C and LDL-C were measured with HITACHI-020 Automatic Biochemical Analyzer. TC/HDL-C, TG/HDL-C, LDL-C/HDL-C, LCI $=\mathrm{TC} \times \mathrm{TG} \times$ LDL-C/HDL-C and non-HDL-C = TC-HDL-C were calculated. CysC and Hcy were measured by AXSYM
Automatic Rapid Immunoassay provided by Abbott USA.

\subsubsection{Normal Reference Interval and Diagnostic Criteria}

Due to regional differences, as well as instruments, reagents and other factors, the hospital reference value range is different. Our hospital $\mathrm{TH}$ normal reference range are as follows: TSH 0.27 4.2mIU/L, FT3 2.8 7.1 pmol/L, FT4 12 22 $\mathrm{pmol} / \mathrm{L}$, T3 $1.3 \sim 3.10 \mathrm{nmol} / \mathrm{L}$, T4 $66 \sim 181 \mathrm{nmol} / \mathrm{L}$; Normal reference range of blood lipid and sugar: FBG 3.89 6.1 $\mathrm{mmol} / \mathrm{L}$, TG $0.56 \sim 1.71 \mathrm{mmol} / \mathrm{L}, \quad$ TC $3.1 \sim 5.17$ $\mathrm{mmol} / \mathrm{L}$, HDL-C 1.09 2.28 mmol/L, LDL-C $<3.36 \mathrm{mmol} / \mathrm{L}$, CysC 0 1.7 mg/L, Hcy 4 15.4ìmol/L. The diagnostic criteria are as follows: Hyperthyroidism has corresponding symptoms or signs and $\mathrm{TSH}<0.27 \mathrm{mIU} / \mathrm{L}, \mathrm{FT} 4>22 \mathrm{pmol} / \mathrm{L}$ and/or FT3 $>7.1$ pmol/L; Hypothyroidism has corresponding symptom or sign and TSH $>4.20 \mathrm{mIU} / \mathrm{L}$, FT $4<12 \mathrm{pmol} / \mathrm{L}$ and/or FT3 $<3.10 \mathrm{pmol} / \mathrm{L}$.

\subsection{Statistical Processing}

Using SPSS 18.0 statistical software for data processing. The measurement data is expressed as mean \pm standard deviation $(\bar{x} \pm \mathrm{s})$. Comparison of single factor analysis of variance. The correlation among $\mathrm{TH}$ and blood lipid profile, blood lipid ratio, CysC and Hcy is analyzed by linear correlation analysis and $\mathrm{P}<0.05$ is statistically significant.

\section{Results}

\subsection{The Comparison of Thyroid Hormone Levels among Groups}

The TSH of hyperthyroidism group is significantly lower than that of control group $(\mathrm{P}<0.001)$. The levels of FT3, FT4, $\mathrm{T} 3$ and T4 in hyperthyroidism group are significantly higher than those in control group $(\mathrm{P}<0.01)$. The TSH of hypothyroidism is significantly higher than that of the control group $(\mathrm{P}<0.001)$. FT3, FT4 and T4 are lower than those of the control group, while T3 and control group have not statistically significant $(\mathrm{P}>0.05)$. See Table 1 .

Table 1. The Comparison of Thyroid Hormone Levels among groups $(\bar{x} \pm s)$.

\begin{tabular}{|c|c|c|c|c|c|c|}
\hline Groups & $\mathbf{n}$ & TSH (mIU/L) & FT3 (pmol/L) & FT4 (pmol/L) & T3 (pmol/L) & T4 (pmol/L) \\
\hline Hyperthyroidism & 100 & $0.128 \pm 0.0246^{\mathbf{\Lambda}}$ & $19.28 \pm 11.22 *$ & $50.46 \pm 24.803 *$ & $5.26 \pm 2.27 *$ & $208.3 \pm 59.94 *$ \\
\hline Hypothyroidism & 100 & $33.2 \pm 12.56 * *$ & $3.61 \pm 1.13^{*}$ & $8.28 \pm 3.23^{*}$ & $1.68 \pm 0.54$ & $96.81 \pm 25.8$ \\
\hline Control & 100 & $2.323 \pm 1.018$ & $4.87 \pm 0.66$ & $18.64 \pm 7.12$ & $1.89 \pm 0.33$ & $105.41 \pm 19.81$ \\
\hline
\end{tabular}

Note: Compared with the normal control group, ${ }^{*} \mathrm{P}<0.05, * * \mathrm{P}<0.01,{ }^{\mathbf{\Delta}} \mathrm{P}<0.001$.

\subsection{The Comparison of Blood Glucose, Blood Lipid Spectrum (mmol/L), CysC and Hcy among Groups $(\bar{x} \pm s)$}

TC, TG, LDL-C, HDL-C of Hyperthyroidism group were significantly lower than the control group $(\mathrm{P}<0.01)$ and Hcy is lower than the control group, but there is no significant difference between the two groups. The FPG, CysC is significantly higher than the control group. TC, TG, LDL-C and Hcy of hypothyroidism group are significantly higher than the control group $(\mathrm{P}<0.05)$, but HDL-C and CysC are lower than the control group. See the Table 2. 
Table 2. The Comparison of blood lipid spectrum, CysC and Hcy among groups $(\bar{x} \pm s)$.

\begin{tabular}{lllllllll}
\hline Groups & $\mathbf{n}$ & FPG & TC & TG & LDL-C & HDL-C & CysC $(\mathbf{m g} / \mathbf{L})$ & Hcy $(\boldsymbol{\mu m o l} / \mathbf{L})$ \\
\hline Hyperthyroidism & 100 & $5.69 \pm 0.63^{*}$ & $3.63 \pm 0.78^{*}$ & $1.09 \pm 0.59^{*}$ & $1.89 \pm 0.59^{*}$ & $1.13 \pm 0.26^{*}$ & $1.48 \pm 0.39^{* *}$ & $12.53 \pm 8.54$ \\
Hypoyhtroidism & 100 & $3.78 \pm 0.88^{*}$ & $5.43 \pm 1.62^{*}$ & $1.97 \pm 1.58^{*}$ & $3.91 \pm 1.14^{*}$ & $1.159 \pm 0.42^{*}$ & $0.78 \pm 0.22$ & $17.98 \pm 37 . *$ \\
Control & 100 & $4.65 \pm 0.91$ & $4.97 \pm 0.96$ & $1.58 \pm 1.20$ & $3.13 \pm 0.38$ & $1.37 \pm 0.33$ & $0.88 \pm 0.18$ & $13.27 \pm 7.75$ \\
\hline
\end{tabular}

Note: Compared with the normal control group, ${ }^{*} \mathrm{P}<0.05$, $* * \mathrm{P}<0.01$.

\subsection{The Comparison of Blood Lipid Ratio, LCI and non-HDL-C among Groups}

TC/HDL-C, TG/HDL-C, LDL-C / HDL-C, LCI, and non-HDL-C of hyperthyroidism are significantly lower than the control group. TG / HDL-C, LDL-C / HDL-C, LCI and
non-HDL-C of hypothyroidism are higher than those of the control group $(\mathrm{P}<0.05)$, but there is no significant difference between TC / HDL-C and control group ( $\mathrm{P}>0.05)$. The results are shown in Table 3.

Table 3. The Comparison of blood lipid ratio and non-HDL-C among groups.

\begin{tabular}{|c|c|c|c|c|c|c|}
\hline Groups & $\mathbf{n}$ & TC/HDL-C & TG/HDL-C & LDL-C/HDL-C & LCI & non-HDL-C \\
\hline Hyperthyroidism & 100 & $3.57 \pm 0.77 *$ & $1.08 \pm 0.596 *$ & $1.23 \pm 0.25 *$ & $1.93 \pm 0.58 * *$ & $2.32 \pm 0.79 *$ \\
\hline Hypothyroidism & 100 & $3.89 \pm 1.17$ & $1.53 \pm 1.42 *$ & $2.90 \pm 0.93 *$ & $33.93 \pm 47.2 *$ & $4.01 \pm 1.39 *$ \\
\hline Control & 100 & $3.82 \pm 1.03$ & $1.32 \pm 1.32$ & $2.42 \pm 0.85$ & $23.43 \pm 29.41$ & $3.60 \pm 0.94$ \\
\hline
\end{tabular}

Note: Compared with the normal control group, $* P<0.05, * * \mathrm{P}<0.01$.

\subsection{The Analysis of the Correlation of Thyroid Hormone and Blood Glucose, Blood Lipid Ratio, non-HDL-C, Hcy and CysC among Groups}

TSH and TC, TG, LDL C, HDL - C, TC/HDL - C, TG/HDL - C, LDL - C/HDL - C, LCI, non - HDL - C, Hcy are positive correlation. FT3, FT4, T3, T4 are positive correlation with FPG, CysC and are negative correlation with TC, TG, HDL- C, LDL- C, TC / HDL-C, TG / HDL-C, LDL-C / HDL-C, LCI, non-HDL-C, CysC. Comprehensive analysis of the results, the correlation of all kinds of thyroid hormones and blood lipid ratio and non-HDL-C, TC is the highest, respectively followed by non-HDL-C, LDL-C and LCI.

\section{Discussions}

The effect of TH on glucose metabolism depends on the level of TH. Most studies have shown that PBG of patients with hyperthyroidism increases and hypothyroidism decreases. Subclinical hyperthyroidism and subclinical hypothyroidism did not change significantly [5-7]. This study is consistent with the above results. Xiaoyan Pan et al [8] found that the levels of blood glucose (PBG1, PBG2) after the meal one hour and two hour in hyperthyroidism group were significantly higher than the control group, and PBG1 was prominent. FBG, PBG1 and PBG2 of hypothyroidism group had a decreasing tendency compared with the control group. But the difference has not statistically significant. There were also reports about hyperthyroidism and hypothyroidism in patients with blood glucose levels and the control group having no statistically significant. It may be related to the selection of cases and the severity of the disease and need further study to be confirmed.

$\mathrm{TH}$ can accelerate the mobilization and decomposition of blood lipids and promote the degradation and excretion of cholesterol, leading to the decrease of total cholesterol levels. However, literature showed varied reports regarding the changes of blood lipids in patients with hyperthyroidism.
Selim et al [9] reported that TC and LDL-C decreased and FT3 and FT4 of patients with hyperthyroidism were significantly negatively correlated with TC, LDL-C and HDL-C and significantly positively correlated with TG; TSH was significantly correlated with TC, LDL-C and HDL-C and significantly negatively correlated with TG. Yan Wang et al [10] did research in 216 cases of hyperthyroidism in patients with lipid found that TG, TC, HDL-C, VLDL-C of hyperthyroidism group were significantly lower than the control group, while LDL-C, T3, T4, FT3, FT4, TG-Ab, Thyroid peroxidase-Ab (TPO-Ab) were higher than the control group. Lin Xiao et al [11] reported that the levels of TC, TG, HDL-C and LDL-C in hyperthyroidism group were significantly lower than the control group. FT3 and FT4 significantly decreased after treatment. TSH and TC, TG, HDL-C and LDL-C were higher than before treatment. Jihong Sun [12] found that FT3 and FT4 of hyperthyroidism patients were higher, while the decrease of TC, TG, LDL-C, Apolipoprotein E (Apo E) was more prominent. FT3 was negatively connected with TC, HDL-C, LDL-C, Apo A1. FT4 was negatively connected with TC, TG, HDL-C, LDL-C and Apo A1. FT4 had the highest correlation with TC $(\mathrm{r}=-0.498$, $\mathrm{P}=0.000)$. Qing $\mathrm{Gu}$ et al [13] reported that TC, TG, HDL-C, LDL-C, ApoB and LP (a) in hyperthyroidism group were lower than the control group. The results of this study showed that the levels of TC, TG, HDL-C and LDL-C in patients with hyperthyroidism were significantly lower than those in the control group $(\mathrm{P}<0.01)$. In addition to the HDL-C in patients with hyperthyroidism was significant higher than that in the control group, which was consistent with the above results. The decrease of TC, TG and LDL-C in patients with hyperthyroidism may be related to the following factors: (1) $\mathrm{TH}$ can accelerate the conversion of LDL-C, increase the number and activity of LDL receptors in liver and promote LDL-C clearance, and reduce the level of LDL-C; (2) TH promotes the use of cholesterol in peripheral tissues, accelerates the excretion of bile acids and bile, and reduces 
blood TC; (3) TH enhances the activity of lipoprotein lipase, accelerates the use of TC in peripheral tissues, and cleanses the clearance rate of $\mathrm{TC}$, which results in decreasing levels of blood TC and TG; (4) TH can promote the reverse transport of cholesterol, accelerate the conversion of cholesterol ester from HDL to LDL, and directly or indirectly reduce TC, TG, HDL-C, and LDL-C. However, it is reported that some patients with hyperthyroidism may have significantly higher TG and LDL-C than those in the control group [14]. Therefore, further in-depth study is required for the changes of blood lipid profile and its mechanism in patients with hyperthyroidism.

For the first time since 1930, Mason, etc. [15] in the NEJM reported "Blood Cholesterol Values in Hyperthyroidism and Hypothyroidism Their Significance", the research of relationship between hypothyroidism and lipid metabolism has received more attention. Garduno GJ et al. [16] found that normal higher TSH and lower TH were associated with blood lipids and were positively correlated with TC, LDL-C and TG and negatively correlated with HDL-C [17]. Fang Li [18] found that TG, TC, and LDL - C of hypothyroidism patients was significantly higher than the control group an. Qingzhang Song et al [19] reported that TG, TC, LDL-C of hypothyroidism's patients significantly increased, while HDL-C significantly decreased. Toruner et al [20] found Subclinical Hypothyroidism $(\mathrm{SCH})$ patients regardless of $\mathrm{TSH} \geq 10 \mathrm{mIU} / \mathrm{mL}$, or $<10 \mathrm{mIU} / \mathrm{mL}, \mathrm{TC}$, TG and LDL-C significantly increased. The study found that TC, TG, LDL-C of hypothyroidism patients were significantly higher than the control group $(\mathrm{P}<0.05)$, while HDL-D decreased. It is the same as the above results.

Blood lipid measurement is a clinical evaluation of the risk of CHD and prognosis of the commonly used indicators. TC / HDL-C, LDL / HDL-C are more valuable than CHD for the diagnosis of CHD [21]. TC / HDL-C and LDL-C / HDL-C can accurately reflect the comprehensive level of lipid metabolism and the risk of CHD in vivo. LDL-C / HDL-C predicts carotid atherosclerotic plaque ratio TC / HDL- $\mathrm{C}$ is more valuable [22]. Yingjun Xie [23] reported that LCI in the CHD group was significantly higher than the control group and the LCI in the unstable angina pectoris (UAP) and acute myocardial infarction (AMI) group was higher than that in the stable angina pectoris group (SAP), while the LCI of UAP and AMI group had no statistical difference. He recognized that the LCI was a good indicator of the risk of CHD. Non-HDL-C represented the total amount of atherosclerotic cholesterol that could accurately reflect the cholesterol levels of atherosclerotic lipoprotein particles, and the predictive and prognostic evaluation of CHD was superior to LDL-C [24]. Xiujuan Chen et al [25] found that non-HDL-C can be used as a supplement to LDL-C, which was to assess the severity of coronary artery disease, recurrent cardiovascular risk and cardiovascular residual risk of new indicators. The results showed that TC / HDL-C, TG / HDL-C, LDL-C / HDL-C, LCI, and non-HDL-C of hyperthyroidism group were significantly lower than the control group $(\mathrm{P}<0.001)$. TG/ HDL-C, LDL-C / HDL-C, LCI and non-HDL-C of hypothyroidism group were significantly higher than the control group $(\mathrm{P}<0.05)$. The correlation between $\mathrm{TH}$ and lipid ratio was the highest with TC, followed by non-HDL-C, LDL-C and LCI, respectively. Suggesting that changes in blood lipid ratio in the abnormal thyroid function than the single indicator was more obvious. TG / HDL-C, LCI and non-HDL-C were calculated to be convenient and not affected by diet and sex, which was to open up new ideas for predicting the occurrence of thyroid dysfunction.

Some studies [26-28] showed that changes in TH levels could cause changes in $\mathrm{CysC}, \mathrm{Cys} \mathrm{C}$ of hyperthyroidism patients was higher than the control group. Meta-analysis of 520 patients with serum $\mathrm{Cys} C$ and thyroid function found that CysC patients of hyperthyroidism significantly increased and hypothyroidism significantly reduced. The results of this study showed that CysC of hyperthyroidism group was significantly higher than the control group and $\mathrm{CysC}$ of hypothyroidism significantly reduced. It was consistent with the above findings, confirming close relation between CysC and thyroid function. However, the mechanism of elevated $\mathrm{CysC}$ in patients with hyperthyroidism was not clear. The study found that $[29,30] \mathrm{TH}$ can stimulate secretion of CysC and impact on CysC. Hyperthyroidism patients with strong basal metabolism, cell renewal rate / metabolic rate to speed up, to promote nucleated cells to produce and secrete CysC faster, may be the direct cause of increase of CysC [31]. However, CysC and TH of patients with hyperthyroidism have the same effect on all nucleated cells, which need further study to be confirmed. Most scholars believed that, CysC was positively correlated with the severity of coronary lesions [32]. Xiaojing Jiang et al [33] found that the higher the serum CysC, the higher risk of the degree of coronary atherosclerosis and instability of multivessel disease and plaque. Shikui Guan et al [34] Meta-analysis of CysC and CHD in 977 patients found that $\mathrm{CysC}$ of $\mathrm{CHD}$ patients significantly increased. It was believed that high levels of $\mathrm{Cys} C$ were closely related to $\mathrm{CHD}$. CysC of hyperthyroidism patients was significantly higher. Therefore, the dynamic monitoring of $\mathrm{Cys} C$ may provide an important basis for the prediction of hyperthyroidism complicated with CHD.

Hyperhomocysteinemia is associated with the onset of CHD. Each increasing of Hcy $4 \mu \mathrm{mol} / \mathrm{L}$ can increase the risk of CHD $4 \%$ and increased Hcy $5 \mu \mathrm{mol} / \mathrm{L}$ and TC increased by $0.5 \mathrm{mmol} / \mathrm{L}$ to increase the incidence of CHD equally [35]. Song Qingzhang et al [19] found that Hcy of hypothyroidism patients was significantly negatively correlated with FT3, FT4, and was significantly positively correlated with TC, and was not correlation with TC, HDL-C, LDL-C. He also recognized that the increasing of serum Hcy was important risk factors of hypothyroidism concurrent CHD. Jihong Sun et al [12] found that FT3 of hypothyroidism negatively related to TC, LDL-C and FT4 negatively related to TC, HDL-C and LDL-C. Wenzhu Yu, et al [36] reported that TG, TC, LDL-C and Hcy of hypothyroidism and $\mathrm{SCH}$ significantly increased and positively correlated with TSH and decreased HDL-C. Chunjuan Zhou et al [4] found that Hcy positively correlated with TSH during SCH transmission to Hypothyroidism. Hcy 
of patients of hypothyroidism elevated indicating a higher incidence of CVD, so combing detection of TSH with Hcy is of great value on the diagnosis and treatment of hypothyroidism. Lu Xiong [37] found that slightly increasing of TSH $(<10 \mathrm{mIU} / \mathrm{L})$ had little effect on TG, LDL-C, but can increase the Hcy and TSH ( $\geq 10 \mathrm{mIU} / \mathrm{L}$ ). It also can make TG, LDL-C, Hcy significantly increase. A meta-analysis showed that Hcy was associated with a severity of hypothyroidism and that treatment with L-T4 can reduce Hcy [38]. This study found that Hcy of hypothyroidism and TSH patients was significantly higher than the control group and the difference between the two groups was statistically significant $(\mathrm{P}<0.05)$, which consistent with the above findings. And TSH positively correlated with TC, TG, LDL-D, TC / HDL-C, TG / HDL-C, LDL-C / HDL-C, LCI, non-HDL-C and Hcy. Increase of Hcy helped predict hypothyroidism with concurrent CHD.

\section{Conclusions}

All in all, the change of thyroid dysfunction in patients with $\mathrm{TH}$ has obvious influence on blood glucose, blood lipid, CysC and Hcy. The changes in blood lipid ratio were more pronounced than those of single index. After treatment, with thyroid function improving, blood lipid profile, blood lipid ratio and Hcy can return to normal. Changes in blood lipid ratio and increasing of CysC and Hcy are risk factors for CHD. Therefore, the dynamic monitoring of thyroid dysfunction in patients with lipid ratio and $\mathrm{CysC}$, Hcy contributes to transmission subclinical thyroid dysfunction to thyroid dysfunction and prediction of thyroid dysfunction recurrent CHD. Joint detection may open up new ways for the prevention and treatment of thyroid dysfunction combined with CVD.

\section{Acknowledgements}

This study was supported by a project grant from: The Science and Technology Plan Project in Hebei Province (Grant No. 15277796D).

\section{References}

[1] Hou ZJ, Zhang JY, Mu ZX, et al. Screening analysis of positive thyroid autoantibody in waterborne high iodine area healthy people. Chinese Journal of Immunology, 2017, vol. 33, pp. 401-406.

[2] Virgini VS, Wijsman LW, Rodondi N, et al. Subclinical thyroid dysfunction and functional capacity among elderly. Thyroid, 2014, vol. 24, pp. 208-214.

[3] Hou ZJ, Hou JZ, Wang FL. Progress of Thyroid Diseases and Cardiovascular Disease Research. Medical Recapitulate, 2015, vol. 21, pp. 4296-4298.

[4] Zhou CJ, Zhu XL, Wang CG, et al. The role of the combined detection of serum thyroid hormone and homocysteine in the diagnosis of hypothyroidism. Chinese Journal of Health Laboratory Technology, 2016, vol. 26, pp. 2205-2207.

[5] Yang XD. Clinical study of abnormal metabolism of blood glucose and blood-fat in patients with hyperthyroidism and hypothyroidism. China Practical Medical, 2015, vol. 10, pp. 58-59.

[6] Xu WD. Analysis of 112 hyperthyroidism patients complicated with metabolic disorder sugar and ester. China Tropical Medicine, 2009, vol. 9, pp. 1725, 1792.

[7] Chen SM. The change and affecting factors of blood lipid and glucose level in pa-tients with subclinical thyroid dysfunction. China Modern Medicine, 2016, vol. 23, pp. 111-114.

[8] Pan XY, Gu XM, Gong XH, et al. Effect of thyroid function on glycolipid metabolism. Journal of Wenzhou Medical University, 2011, vol. 41, pp. 274-276.

[9] Selim FO, Ahmed AM. The association between serum paraxonase-1 activity, thyroid hormones and lipids profile in patients with primary hyperthyroidism. Int J Adv Res, 2014, vol. 2, pp. 172-181.

[10] Wang Y, Ruan HL, Li Y, et al. Changes of thyroid function, autoantibodies, bone mineral density and bone metabolism indexes in patients with hyperthyroidism. Journal of Hainan Medical University, 2016, vol. 22, pp. 1418-1420.

[11] Xiao L, Guo M, Fan RY. Study on the Relationship between Serum Lipid Metabolism and Thyroid Hormone Levels in Patients with Hyperthyroidism. Chinese Journal of Laboratory Diagnosis, 2016, vol. 20, pp. 1679-1681.

[12] Sun JH, Zhang Y, Lu HR, et al. Correlation of serum adiponectin with thyroid dysfunction in elderly people. Laboratory Medicine, 2016, vol. 31, pp. 549-552.

[13] Gu Q, Shao KK, Wang YX. Analysis of blood lipid and serum protein levels in patients with abnormal thyroid function. Laboratory Medicine and Clinic, 2014, vol. 11, pp. 3455-3456.

[14] Zhang ZZ, Zhang HM, Fu XL. The relationship of the levels of thyroid hormone and atherosclerosis related factors of patients with hyperthyroidism. Chinese Journal of Difficult and Complicated Cases, 2015, vol. 15, pp. 926-930.

[15] Zhao JJ, Yang LB. Hypothyroidism and dyslipidemia. Chinese Journal of Practical Internal Medicine, 2014, vol. 34, pp. 340-343.

[16] Garduno-Garcia Jde J, Alvirde-Garcia U, Lopez-Carrasco G, et al. TSH and free thyroxine concentrations are associated with differing metabolic markers in euthyroid subjects. Eur J Endocrinol, 2010, vol. 163, pp. 273-278.

[17] Fu YL, Fan LM. Progress in the study of the correlation between thyroid function and metabolic syndrome. Journal of Modern Medicine \& Health, 2017, vol. 33, pp. 77-79.

[18] Li F. The test and clinical significance of serum homocysteine and blood lipids in subclinical hypothyroidisn. Zhejiang Practical Medicine, 2009, vol. 14, pp. 366-367.

[19] Song QZ, Xu FL, Bi XP, et al. Clinical significance of serum homocysteine and lipids determination in hypothyroidism. Laboratory Medicine, 2007, vol. 22, pp. 176-178.

[20] Toruner F, Altinova AE, Karakoc A, et al. Risk factors for cardiovascular disease in patients with subclinical hypothyroidism. Adv Ther, 2008, vol. 25, pp. 430 437.

[21] Wang C, Zhang P. Clinical research progress of blood lipid ratio in predicting cardiovascular disease. Shandong Medical Journal, 2015, vol. 55, pp. 99-102. 
[22] Huang X, Cui ZW, Wu W, et al. The application of blood lipid ratio in the risk assessment of cardiovascular and cerebrovascular diseases. International Journal of Laboratory Medicine, 2015, vol. 36, pp. 1274-1276.

[23] Xie YJ, The level and significance of blood lipid synthesis index in patients with different types of coronary heart disease. Contemporary Medicine, 2012, vol. 18, pp. 10-11.

[24] Simprini LA, Villines TC, Rich M, et al. The relationship between subclinical atherosclerosis, non-high-density lipoprotein cholesterol, exercise and diet among male participants of the PACC project. J Clin Lipidol, 2012, vol. 6, pp. 174-179.

[25] Chen XJ, Huang ZH. The value of non-high density lipoprotein cholesterol in predicting and evaluating the prognosis of coronary atherosclerotic heart disease. China Medical Engineering, 2016, vol. 24, pp. 53-55.

[26] Ye Y, Gai X, Xie H, Jiao L, Zhang S. Impact of thyroid function on senlm cystatin $\mathrm{C}$ and estimated glomerular filtration rate: a cross-sectional study. Endocr Pract, 2013, vol. 19, pp. 397-403.

[27] Xu YQ. Application of combined detection of serum cystatin C, creatinine and beta 2- microglobulin in thyroid dysfunction. The Journal of Medical Theory and Practice, 2015, vol. 28, pp. 953-954.

[28] Yu WG, Liu Z, Zhang DF, et al. Meta-analysis of Relationship Between Serum Cystatin C Levels and Thyroid Function. Labeled Immunoassays and Clinical Medicine, 2016, vol. 23, pp. 579-582, 589 .

[29] Schmid C, Ghirlanda-Keller C, Zwimpfer C, et al. Triiodothyronine stimulates cystatin $\mathrm{C}$ production in bone cells. Biochem Biophys Res Commun, 2012, vol. 419, pp. 425-430.

[30] Wang F, Pan W, Wang H, et al. The impacts of thyroid function on the diagnostic accuracy of cystatin $\mathrm{C}$ to detect acute kidney injury in ICU patients: a prospective, observational study. Crit Care, 2014, vol. 18, pp. R9.

[31] Fricker $M$, Wiesli $P$, Brändle $M$, et al. Impact of thyroid dysfunction on serum cystatin C. Kidney Int, 2003, vol. 63, pp. 1944-1947.

[32] Zhang D, Wang CX. Research progress of cystatin C in the clinical application of coronary heart disease. Medical Journal of West China, 2014, vol. 26, pp. 127-128, 131.

[33] Jiang XJ, Chen XY, Chen H, et al. Effect of serum cystatin C on status of coronary atherosclerotic plaques. Practical Journal of Clinical Medicine, 2016, vol. 13, pp. 99-101.

[34] Guan SK, Liu Z, Zhang FS, et al. A Meta-analysis on Relationship between Serum Cystatin C Level and Coronary Heart Disease. Labeled Immunoassays and Clinical Medicine, 2015, vol. 22, pp. 994-997.

[35] Wu ZR, Zhang LR, Pan YQ, et al. Changes and clinical significance of serum homocysteine and lipids in Patients with primary hypothyroidism and hyperthyroid. Progress in Modern Biomedicine, 2009, vol. 9, pp. 4276-4278.

[36] Yu WZ, Yuan D, Fan YX, et al. Correlation analysis of blood lipid, Homocysteine and Uric acid of patients with subclinical hypothyroidism. Chinese Journal of Laboratory Diagnosis, 2015, vol. 19, pp. 1496-1497.

[37] Xiong L, Luo CD, Liu YB, et al. Changes of plasma homocysteine and peripheral arterial stiffness in subclinical hypothyroidism. West China Medical Journal, 2015, vol. 30, pp. 2205-2207.

[38] Zhou Y, Chen Y, Cao X. Association between plasma homocysteine status and hypothyroidism: a meta- analysis. Int J Clin ExpMed, 2014, vol. 7, pp. 4544-4553. 